\title{
Isostatic Recovery and the Strain Rate Dependent Viscosity of the Earth's Mantle
}

\author{
Christopher Brennen
}

Hydrodynamics Laboratory, California Institute of Technology, Pasadena, California 91109

\begin{abstract}
This paper is concerned with the interpretation of isostatic recovery data in terms of the flow properties of the earth's mantle. A hydrodynamic analysis is first presented that allows straightforward calculation of the relaxation time for isostatic recovery within a mantle in which the viscosity varies continuously with depth. However, it transpires that no curve of this type (i.e., choice of a reference viscosity and a rate of change of viscosity with depth) can of itself adequately explain the available observational data from the Fennoscandian and Laurentide ice sheets and the pluvial Lake Bonneville. Proceeding onward it is then demonstrated that the strain rates within such flows are in fact greater than the critical strain rate envisaged by Weertman (1970) in his theoretical rheological model of the mantle. Below this critical value, diffusion creep is the dominant flow process, and the flow can be modeled by a Newtonian viscosity. But above this value, dislocation glide takes over, and the viscosity exhibits a decrease with increasing strain rate. This feature is then incorporated into the theoretical model, and the isostatic recovery data are interpreted in such a way as to provide experimental values of the strain rate dependent viscosity that can be compared with the values in Weertman's rheological model. It is demonstrated that the data become most self-consistent and exhibit the most satisfactory agreement with Weertman's model when the increase of mantle viscosity with depth is given roughly by $\exp \left(5 \times 10^{-4} z\right)$, where $z$ is the depth in kilometers. Thus in addition, the analysis would appear to provide some verification of Weertman's model of the mantle flow properties. It is further demonstrated that the much larger increase of viscosity with depth predicted by McConnell (1968) and others from previoùs analyses of isostatic recovery data is an artifice induced by the nature of such flows in which the strain rate decreases with depth; this led to an apparent increase of viscosity that is much larger than the actual variation.
\end{abstract}

The conventional concept of the earth's crust envisages a firm outer layer, the lithosphere, which is about $100-300 \mathrm{~km}$ thick and is divided into individual tectonic plates. These plates float on the weaker asthenosphere, or mantle, which extends inward about $3000 \mathrm{~km}$ to a radius of about $3300 \mathrm{~km}$ from the earth's center. Inside of the mantle lies the molten liquid core.

It is now recognized that the flow processes occurring within the mantle itself play a major role in quite a number of geological phenomena. For example, the large temperature differences across the mantle are believed to have established thermal convection cells in the asthenosphere, which are the driving mechanism for crustal warping, orogenics, and continental drift. Further, when the crust is locally relieved of a large mass, such as the ice sheets or pluvial lakes of the Pleistocene, the ice sheets will seek a new buoyancy equilibrium, a process known as isostatic recovery. The relaxation time $T_{R}$ for this process is clearly related to the viscous flow response in the mantle and therefore to its viscosity. Indeed, detailed studies of isostatic recovery have been one of the primary means by which the viscosity of the upper mantle has been estimated.

Early analyses of isostatic recovery were based on the viscous flow solutions of Vening Meinesz [1937], Haskell [1935, 1936, 1937], Niskanen [1948], and Heiskanen and Vening Meinesz [1958], which assumed that the mantle flows like a Newtonian liquid and has a uniform viscosity. For an infinitely deep medium this assumption yielded the relation

$$
T_{R} \propto \mu / \rho g L
$$

where $\mu$ is the dynamic viscosity, $\rho$ is the mean mantle density. $g$ is the gravitational acceleration at the surface, and $L$ is the linear extent of the removed loading. With this in mind

Copyright () 1974 by the American Geophysical Union.
Heiskanen and Vening Meinesz analyzed the isostatic recovery of the Fennoscandian ice sheet [Haskell, 1937; Niskanen, 1948], and Crittenden [1963] did the same for pluvial Lake Bonneville. The two loads differed in linear extent by a factor of about 10 . Thus a dilemma arose when the relaxation times were found to differ only slightly (order of 5000 and 4000 years, respectively). Takeuchi [1963] then suggested that the flow may be concentrated primarily in a relatively thin layer in the upper mantle. Jeffreys's [1952] analysis showed that if a lower horizontal boundary were placed in the flow at a depth $D$, where $D \ll L$, then $T_{R} \propto L^{2}$, as opposed to the $T_{R} \propto L^{-1}$ of (1). But this did not answer the dilemma except by the otherwise unsupported postulation of different thicknesses $D$ of the flowing layer at different places. However, a mantle viscosity that is not uniform but increases with depth would have a similar effect of concentrating the flow in a region near the surface. With this in mind McConnell [1968], Takeuchi and Sakata [1970], and others relaxed the second of the early assumptions, i.e., that the mantle has a uniform viscosity, and attempted from the isostatic recovery data to construct a model of the mantle composed of layers of fluid of different viscosity, predominantly increasing with depth. A few of McConnell's models are indicated in Figure 1.

All of these models presumed as did those of Heiskanen and Vening Meinesz that the mantle flowed like a Newtonian liquid. This assumption was given some credibility by the pioneering theoretical studies of Gordon [1965, 1967], who concluded that the flow process in the mantle was one of diffusion creep (Herring-Nabarro creep), in which the stress is indeed simply proportional to the strain rate. Moreover, the factor of proportionality, or viscosity, is a function of temperature. Hence on the basis of an estimated variation of temperature with depth Gordon predicted a rather dramatic increase of viscosity with depth in the mantle; a curve similar to that of Gordon (though it was actually taken from Weertman 


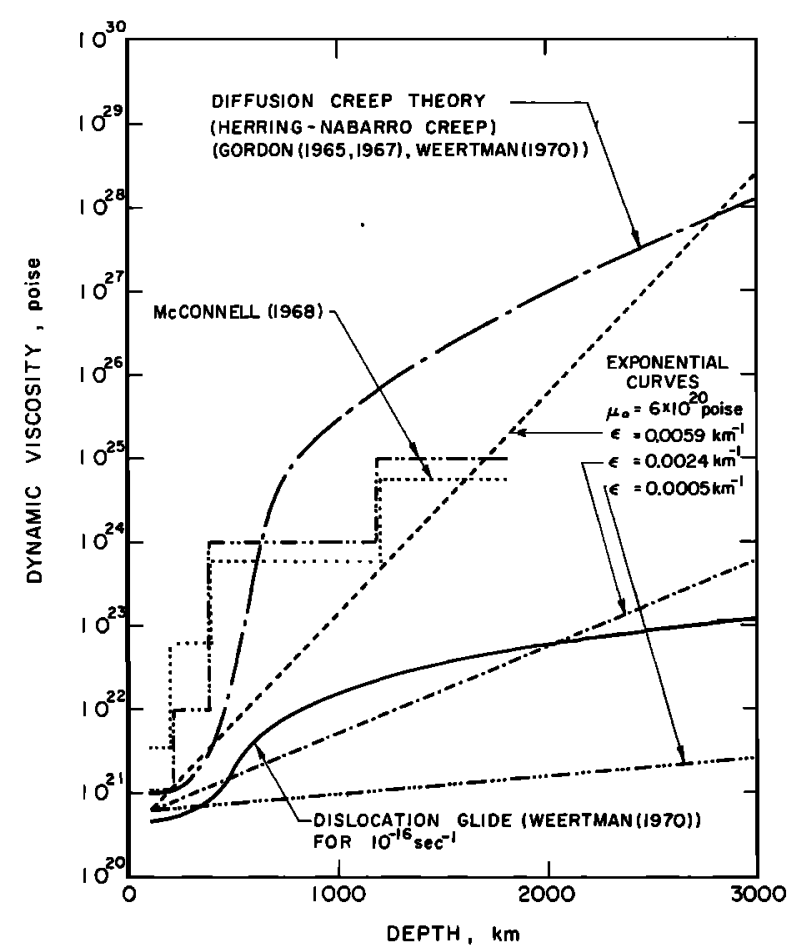

Fig. 1. Viscosity of the earth's mantle as a function of depth: theoretical model based only on diffusion creep (Herring-Nabarro creep [Gordon, 1965, 1967; Weertman, 1970]); Weertman's [1970] theoretical model based on dislocation glide at a shear rate of $10^{-16}$ $\mathrm{s}^{-1}$; exponentially increasing viscosity profiles for $\mu_{0}=0.6 \times 10^{21} \mathrm{P}, \epsilon$ $=0.0059 \mathrm{~km}^{-1}, \epsilon=0.0024 \mathrm{~km}^{-1}$, and $\epsilon=0.0005 \mathrm{~km}^{-1}$; and viscous layer model of McConnell [1968].

[1970]) for a mantle viscosity based on diffusion creep is shown in Figure 1. This is in apparent qualitative agreement with the models of McConnell; it also appeared to yield viscosities of the lower mantle that were in accord with the value of $10^{28} \mathbf{P}$ suggested by Macdonald [1963] on the basis of the response of the earth's shape to the reduction in its rotational velocity.

However, it is now becoming increasingly apparent that such agreement is merely fortuitous. In a more recent and thorough theoretical study of flow processes in the mantle Weertman [1970] concludes that although diffusion creep may be the dominant process at very low strain rates, at higher strain rates the processes of dislocation climb and dislocation glide creep will take over. Since these are non-Newtonian in the sense that the strain rate is proportional to the stress to the third power (for dislocation glide), the viscosity becomes a function of strain rate. Further, the factor of proportionality is a function of temperature or, more precisely, the ratio of actual temperature to melting point temperature $\left(T / T_{M}\right)$. Hence based on a number of best guesses as to grain size etc., Weertman proposes a rheological model for the mantle, which is shown in Figure 2. In addition, given a reasonable temperature profile for the mantle, this model results in a mantle viscosity profile (at a strain rate of $10^{-16} \mathrm{~s}^{-1}$ ) that exhibits (Figure 1) a much smaller increase of viscosity with depth than that based on diffusion creep alone.

But Weertman's viscosity profile based on dislocation glide (Figure 1) is now at odds with the isostatic recovery data and Macdonald's [1963] lower mantle viscosity of $10^{28} \mathrm{P}$. However, with regard to the $10^{28} \mathrm{P}$ value, a more recent analysis of the excessive fossil equatorial bulge by Dicke [1969] yielded a value of $10^{22} \mathrm{P}$ for the lower mantle. In addition, Goldreich and
Toomre [1969] suggest $10^{22} \rightarrow 10^{24} \mathrm{P}$ for the viscosity of the lower mantle in order to explain polar wandering. These values are thus in accord with Weertman's profile.

The intention of this paper is to reexamine the isostatic recovery data in the light of Weertman's model. It will be shown that the rapid increase of viscosity with depth inferred from the isostatic recovery data by McConnell [1968] and others is most probably an artifice caused by the Newtonian assumption. Briefly, the thesis examined in this paper is as follows: The nature of the flow due to isostatic recovery is such that the strain rate decreases fairly rapidly with depth. Moreover, a quantitative evaluation of these strain rates (given in the section on comparison of isostatic recovery data with Weertman's theory) indicates that these are greater than the critical values of Figure 2 . Hence the flow will experience an apparent increase of viscosity with depth simply because of the decreasing strain rate. It will be shown that the models of McConnell (Figure 1) exhibit not a real increase in viscosity but rather this apparent increase; it will further be shown that an analysis of the isostatic recovery data that permits a strain rate dependent viscosity yields mantle viscosity profiles that are consistent with Weertman's theory.

We begin in the next section with the hydrodynamic solution for an isostatic recovery flow in a mantle in which the viscosity varies with depth in the following simple manner:

$$
\mu=\mu_{0} e^{-\epsilon z}
$$

where $\mu_{0}$ is the viscosity immediately below the crust, $(-z)$ is the depth beyond that point, and $\epsilon$ is the exponent of the viscosity variation. Three such profiles are indicated in Figure 1.

\section{Isostatic Recovery Flow in MANTLe of NONUNIFORM VISCOSITY}

Because of mathematical necessity and because of the small size of the amplitudes of the surface wave motion in comparison with the wavelength, it is assumed that the temperature distribution and the viscosity variation are effectively undisturbed by the flow. This assumption may seem contradictory in the light of the earlier discussions, but it is convenient to develop this solution first.

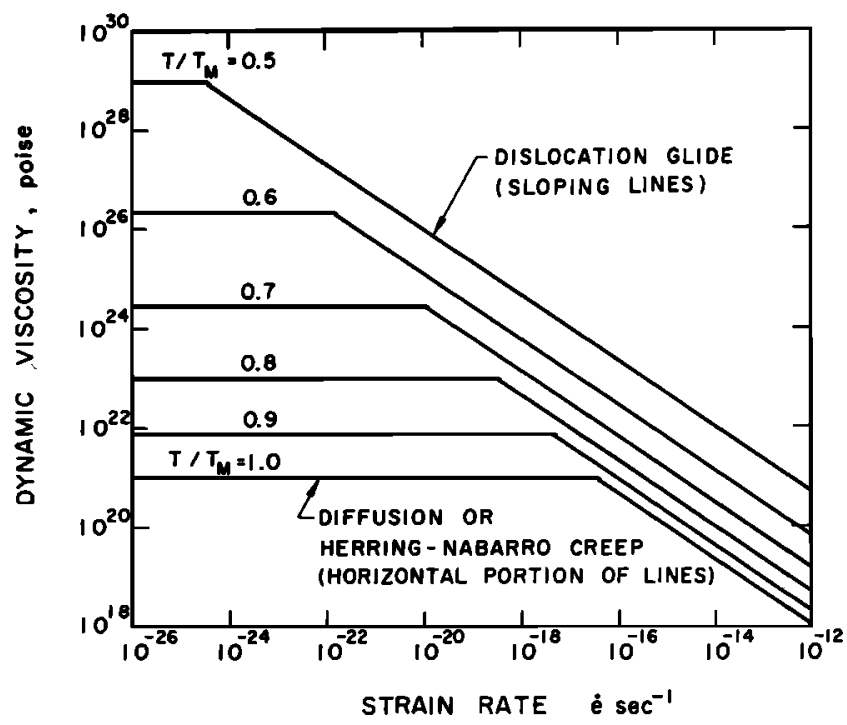

Fig. 2. Variation of the mantle viscosity with strain rate $\dot{e}$, and actual temperature to melting temperature ratio $T / T_{M}$, according to Weertman's [1970] theory. 
The Navier-Stokes equations of motion for a fluid of nonuniform viscosity are [Milne Thomson, 1968]

$$
\begin{aligned}
\rho \frac{D \mathbf{v}}{D t}=\rho \mathrm{F}- & \nabla p-\mu \nabla \times(\nabla \times \mathbf{v}) \\
& +2(\nabla \mu \cdot \nabla) \mathrm{v}+(\nabla \mu) \times(\nabla \times \nabla)
\end{aligned}
$$

where $\mathbf{v}$ is the velocity vector, $\rho$ is the density (assumed to be uniform), $\mathrm{F}$ is the body force vector, $p$ is the pressure, $\mu$ is the dynamic viscosity, and $t$ is time. The equation of continuity is

$$
\nabla \cdot(\rho v)=0
$$

Although it is necessary in studying the stability of the mantle to consider the thermal variation of density through the Boussinesq approximation [Chandrasekhar, 1961], this is not necessary for isostatic recovery flows [Heiskanen and Vening Meinesz, 1958]. It is sufficient to assume that the fluid is incompressible and that the gravitational body force takes its surface value $g$. Since the Reynolds numbers of the motions are extremely small, the inertial terms on the left-hand side of (3) can be neglected. Thus the equations reduce to those of Stokes flow, but for nonuniform viscosity,

$$
\begin{gathered}
\nabla(p+\rho g z)=\mu \nabla \times(\nabla \times v) \\
+2(\nabla \mu \cdot \nabla) v+(\nabla \mu) \times(\nabla \times \nabla)=0 \\
\nabla \cdot \nabla=0
\end{gathered}
$$

where $z$ is the coordinate perpendicular to the surface, positive in the upward direction and zero at a point immediately below the crust.

Two particular geometries, one planar and the other axisymmetric, will be considered simultaneously, since they yield similar results. The planar case corresponds to an unloading along a strip that is infinitely long, so that the flow is planar in the $x z$ plane, $x$ being a horizontal surface coordinate. The axisymmetric case corresponds to the unloading in a circular surface area, so that the flow velocity vector $v$ is a function only of $r, z$, where $(r, \theta)$ are polar coordinates on the surface. Defining a stream function $\psi$ such that

$$
v_{x}=\frac{\partial \psi}{\partial z} \quad v_{z}=-\frac{\partial \psi}{\partial x}
$$

in the planar case, and

$$
v_{r}=\frac{1}{r} \frac{\partial \psi}{\partial z} \quad v_{z}=-\frac{1}{r} \frac{\partial \psi}{\partial r}
$$

in the axisymmetric case, and taking the curl of (5) to eliminate the pressure yields the following differential equation for $\psi$ when $\mu$ is a function only of $z$ :

$$
\mu L_{1}\left[L_{1}(\psi)\right]+2 \frac{\partial \mu}{\partial z} \frac{\partial}{\partial z}\left[L_{1}(\psi)\right]-\frac{\partial^{2} \mu}{\partial z^{2}} L_{2}(\psi)=0
$$

where in the planar case the operators are

$$
L_{1,2}=\frac{\partial^{2}}{\partial x^{2}} \pm \frac{\partial^{2}}{\partial z^{2}}
$$

and in the axisymmetric case the operators are

$$
L_{1,2}=\frac{\partial^{2}}{\partial r^{2}}-\frac{1}{r} \frac{\partial}{\partial r} \pm \frac{\partial^{2}}{\partial z^{2}}
$$

Now by substituting the relation (2) $\left(u=\mu_{0} e^{-\varepsilon z}\right)$ and examining the characteristic harmonic solutions of (9) for which $\psi=$
$A e^{\alpha z} H(x$ or $r)$ and

$$
H(x)=\sin k x
$$

in the planar case and

$$
H(r)=r J_{1}(k r)
$$

in the axisymmetric case, where $A$ is some arbitrary small amplitude, $k$ is a wave number, and $\alpha$ is as yet undetermined, the following dispersion relation is obtained in both cases:

$\alpha^{4}-2 \epsilon \alpha^{3}+\left(\epsilon^{2}-2 k^{2}\right) \alpha^{2}+2 \epsilon k^{2} \alpha+k^{2}\left(k^{2}+\epsilon^{2}\right)=0$

The solution of (14) yields four possible values of $\alpha$ :

$$
\alpha=\frac{\epsilon}{2} \pm\left[\left(\frac{\epsilon}{2}\right)^{2}+k^{2} \pm i k \epsilon\right]^{1 / 2}
$$

To simplify the presentation set

$$
\beta=\left\{1 / 2\left[k^{2}+(\epsilon / 2)^{2}+\left(k^{2} \epsilon^{2}+\left(k^{2}+(\epsilon / 2)^{2}\right)^{2}\right)^{1 / 2}\right]\right\}^{1 / 2}
$$

so that (15) becomes

$$
\alpha=\frac{\epsilon}{2} \pm \beta \pm \frac{i k \epsilon}{2 \beta}
$$

the choices of sign being independent. However, since solutions for which $\alpha$ has a negative real part lead to physically unrealistic solutions with velocities increasing indefinitely with depth, it is only necessary to consider the solutions

$$
\alpha=\left(\frac{\epsilon}{2}+\beta\right) \pm\left\{\frac{i k \epsilon}{2 \beta}\right\}
$$

It follows that

$\psi=A(t) \exp \left[\left(\frac{\epsilon}{2}+\beta\right) z\right] \cos \left\{\frac{k \epsilon}{2 \beta} z+\varphi\right\} H(x$ or $r)$

where $\varphi$ is some as yet undetermined phase angle and the amplitude $A$ may now be regarded as an unknown function of time $t$.

Boundary conditions. Consider the imposition of the surface boundary conditions on this characteristic solution from which more complex solutions may be Fourier-synthesized. Since the horizontal motion of the crust is negligible in comparison with the vertical motions and the vertical displacements are small in comparison with lateral dimensions, a linearized condition on the free surface $z=\eta(x$ or $r, t)$ is quite justified, and the kinematic conditions become

$$
\begin{array}{lll}
v_{x}=0 \text { or } & v_{r}=0 & \text { on } z=0 \\
v_{z}=\frac{\partial \eta}{\partial t} & & \text { on } z=0
\end{array}
$$

It follows from (19) and (20) that

$$
\tan \varphi=\frac{2 \beta}{k \epsilon}\left(\frac{\epsilon}{2}+\beta\right)
$$

And it follows further from (21) and (19) that

$$
\frac{\partial \eta}{\partial t}=-A k H^{\prime} \cos \varphi
$$


where $H^{\prime}$ is $\cos k x$ in the planar case and $J_{0}(k r)$ in the axisymmetric case.

A dynamic free-surface condition that determines the normal stress $\sigma_{z z}$ in the fluid at the surface is also required. If the surface is considered as having been completely unloaded, then the linearized form of this condition is

$$
p=\sigma_{2 z}=0 \text { on } z=\eta
$$

where simplification occurs by use of (20). From the form of (19) and the basic equation (5) this condition yields the surface displacement $\eta$ as

$$
g \rho \eta=-A \mu_{0} H^{\prime} \Sigma / k
$$

where

$$
\begin{aligned}
\Sigma & =\cos \varphi\left[\left(\beta-\frac{\epsilon}{2}\right)\left(\frac{\epsilon}{2}+\beta\right)^{2}\right. \\
& \left.-\left(\frac{k \epsilon}{2 \beta}\right)^{2}\left(\frac{\epsilon}{2}+3 \beta\right)-k^{2}\left(\frac{3 \epsilon}{2}+\beta\right)\right] \\
& -\frac{k \epsilon}{2 \beta} \sin \varphi\left[3\left(\frac{\epsilon}{2}+\beta\right)^{2}-2 \epsilon\left(\frac{\epsilon}{2}+\beta\right)-\left(\frac{k \epsilon}{2 \beta}\right)^{2}-k^{2}\right]
\end{aligned}
$$

Elimination of $A H^{\prime}$ from (23) and (25) then yields the following differential equation for $\eta$ :

$$
\frac{\partial \eta}{\partial t}=-\left[\frac{\rho g}{\mu_{0} K}\right] \eta
$$

where

$$
K=\frac{\epsilon}{k}\left\{1+\frac{1}{2}\left[\frac{\epsilon}{\beta}+\frac{\beta}{\epsilon}\left(\frac{\epsilon}{k}\right)^{2}\left(1+\frac{2 \beta}{\epsilon}\right)^{2}\right]\right\}
$$

The relaxation time $T_{R}$ defined in the manner of Heiskanen

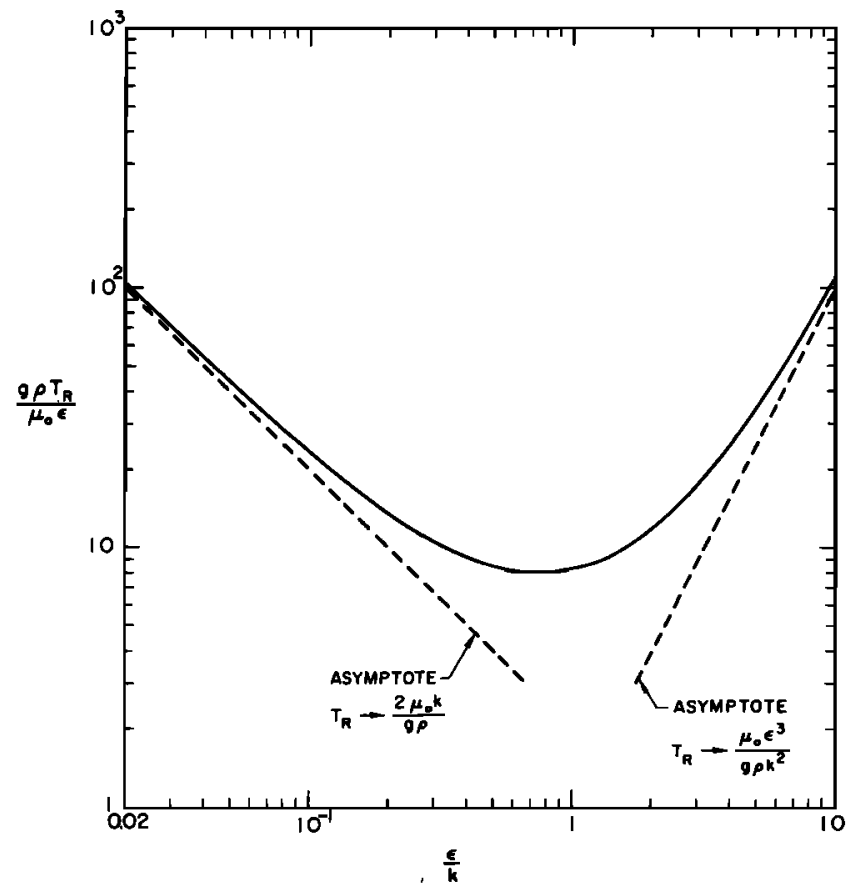

Fig. 3. Theoretical relation between the relaxation time for isostatic recovery $T_{\mathrm{R}}$, the wave number $k$, and the viscosity of a mantle given by $\mu_{0} e^{-e z}$, where $(-z)$ is the depth. and Vening Meinesz [1958] as $T_{R}{ }^{-1}=-\eta^{-1} \partial \eta / \partial t$ follows immediately from (27a) and is given in both the planar and the axisymmetric case by

$$
T_{R}=\mu_{0} k K / \rho g
$$

Given $\epsilon$ and the wave number $k, \rho g T_{R} / \mu_{0}$ may be evaluated directly by using the expression (16) for $\beta$. This evaluation is shown in Figure 3, where $\rho g T_{R} / \mu_{0} \epsilon$ is plotted against $\epsilon / k$. It represents a generalization of earlier analyses to the case of nonuniform viscosity. Note that when $\epsilon / k$ is very small, the curve approaches the asymptote

$$
T_{\mathrm{R}} \rightarrow 2 \mu_{0} k / g \rho
$$

which corresponds to the result of Heiskanen and Vening Meinesz [1958]. On the other hand when $\epsilon / k$ is large, the curve approaches the asymptote

$$
T_{R} \rightarrow \mu_{0} \epsilon^{3} / g \rho k^{2}
$$

which, when $k$ is recognized as representing $1 / L$, where $L$ is the linear extend of the removed load, exhibits the same kind of dependence of $T_{R}$ on $L$ as the solution of Jeffreys [1952] mentioned in the introduction.

Clearly, if the quantities on the right-hand side of (28) are independent of time, $T_{R}$ remains constant throughout the motion, and isostatic recovery is exponential. However, in the section on strain rates in isostatic recovery flows it will be demonstrated that a strain rate dependent viscosity leads to a time dependent $\mu_{0}$ and hence to isostatic recovery that is no longer exponential. Nevertheless, at any instant in this motion, $T_{R}$ is still given by (28).

\section{Isostatic Recovery Data}

The curve of Figure 3 is replotted dimensionally in Figure 4 for a number of different values of $\epsilon$ and $\mu_{0}, \rho$ being taken as $3.4 \mathrm{~g} / \mathrm{cm}^{3}$ and $\mathrm{g}$ as $980 \mathrm{~cm} / \mathrm{s}^{2}$. Also shown are the data of

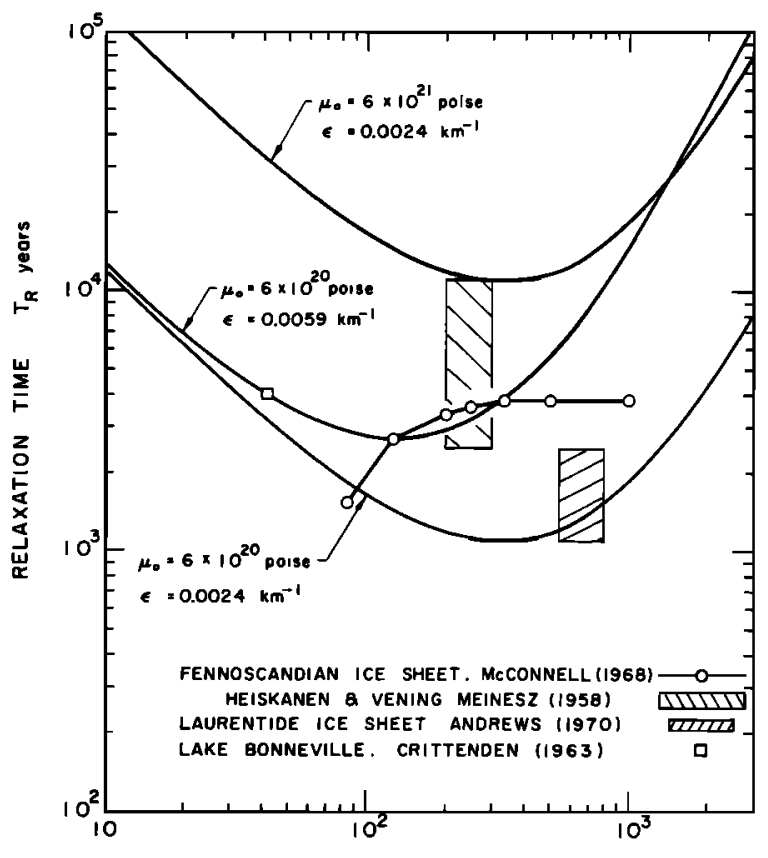

RECIPROCAL OF WAVE NUMBER, $k^{-1} \mathrm{~km}$

Fig. 4. Comparison of relaxation times $T_{R}$ of isostatic recovery with theoretical curves based on Figure 3. 
Heiskanen and Vening Meinesz [1958] and McConnell [1968] for the Fennoscandian ice sheet, the data of Crittenden [1963] for the pluvial Lake Bonneville, and some data taken from $\mathrm{An}$ drews [1970] for the Laurentide ice sheet. The data of McConnell include a Fourier analysis in order to ascertain the relaxation times $T_{R}$ for the different wave numbers that compose the surface motion. The relaxation times given by Heiskanen and Vening Meinesz [1958] are assumed to be relevant to the dominant range of wave numbers in McConnell's analysis. For the rest of the data it was assumed that a wave number comparable to the lateral dimension $L$ would dominate. Since the loads are predominantly axisymmetric and since the first zero of $J_{0}(k r)$ occurs at $k r \approx 2.4$, it was assumed that $k=4.8 / L$, where $L=200 \mathrm{~km}$ for Lake Bonneville [Crittenden, 1963] and around $3400 \mathrm{~km}$ for the Laurentide ice sheet.

McConnell [1968] attempted to fit a layered mantle model to similar observational data and hence arrived at the mantle viscosities plotted in Figure 1. The analysis of the next section attempts to show that such an analysis is inconsistent with Weertman's theory.

However, in passing it might be noted that there is clearly no theoretical curve of the type derived in the last section that could fit all the observational data. It is especially notable that McConnell's own data have an entirely different trend. Furthermore, as is clearly indicated by the values in Table 1 , $T_{R}$ is not independent of time for a particular isostatic recovery motion. In order to reexamine the observational data in the light of Weertman's theory and in the manner suggested at the end of the introduction, the strain rate distribution within an isostatic recovery flow must be examined.

\section{Strain Rates in Isostatic Recovery Flows}

In order to proceed it is necessary to interpret the solution of the section on isostatic recovery flow in mantle of nonuniform viscosity in a different manner. First, note that the shear strain rate of that isostatic recovery flow is given simply by $L_{1}(\psi)$ in the planar case and $L_{1}(\psi) / r$ in the axisymmetric case. It follows from the solution (19) that the strain rate $\dot{e}$ is of order

$$
\dot{e} \simeq\left\{k \frac{\partial \eta}{\partial t}\right\} \exp \left[\left(\frac{\epsilon}{2}+\beta\right) z\right]
$$

The quantitative evaluation of the next section shows that the strain rates given by (31) are sufficiently large for the consequent motions to be governed by Weertman's dislocation glide, so that

$$
\mu=\mu^{*}(\grave{e})^{-2 / 3}
$$

where $\mu^{*}$ is a function of $T / T_{M}$, and since $T / T_{M}$ is a function of depth in the mantle, $\mu^{*}$ may be regarded as a function of $z$ in general. To simplify matters, it will be assumed that this temperature-controlled depth variation can be approximated by

$$
\mu^{*}=\mu_{0}^{*} \exp \left(-\epsilon^{*} z\right)
$$

where $\mu_{0}^{*}$ is a constant and values of $\epsilon^{*}$ in the range $0 \rightarrow 0.006$ $\mathrm{km}^{-1}$ deserve attention in view of the data of Figure 1 . This $\epsilon^{*}$

TABLE 1. Strain Rates $\dot{e}_{0}$ and Viscosities $\mu_{0}$ From the Analysis of Some Isostatic Recovery Data for Different Values of the Real Viscosity Variation $\varepsilon^{*}$

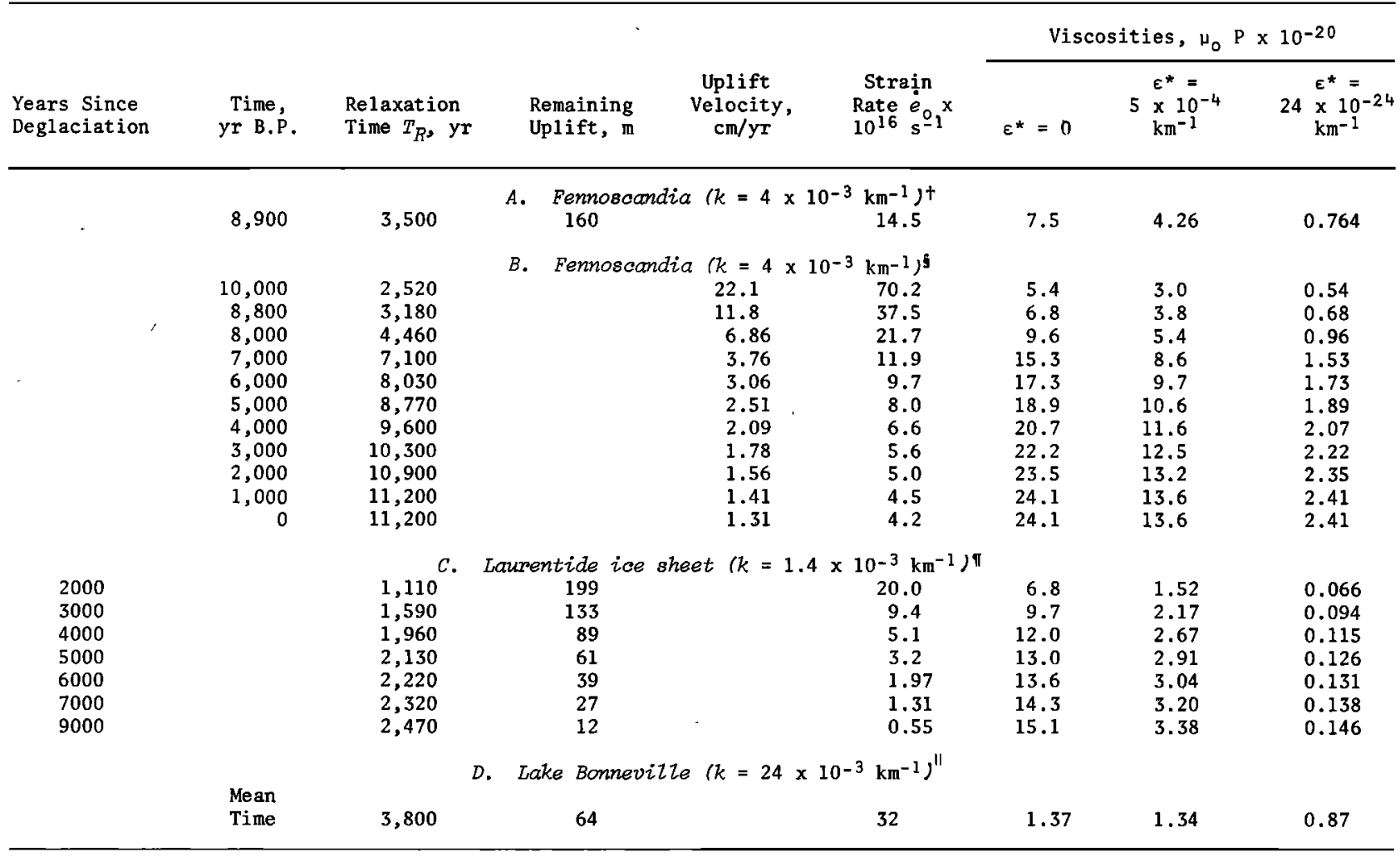

TMcConne 22 [1968].

$\$_{H e i s k a n e n}$ and Vening Meinesz [1958].

"Andrews [1970].

$\|_{\text {Crittenden [1963]. }}$ 


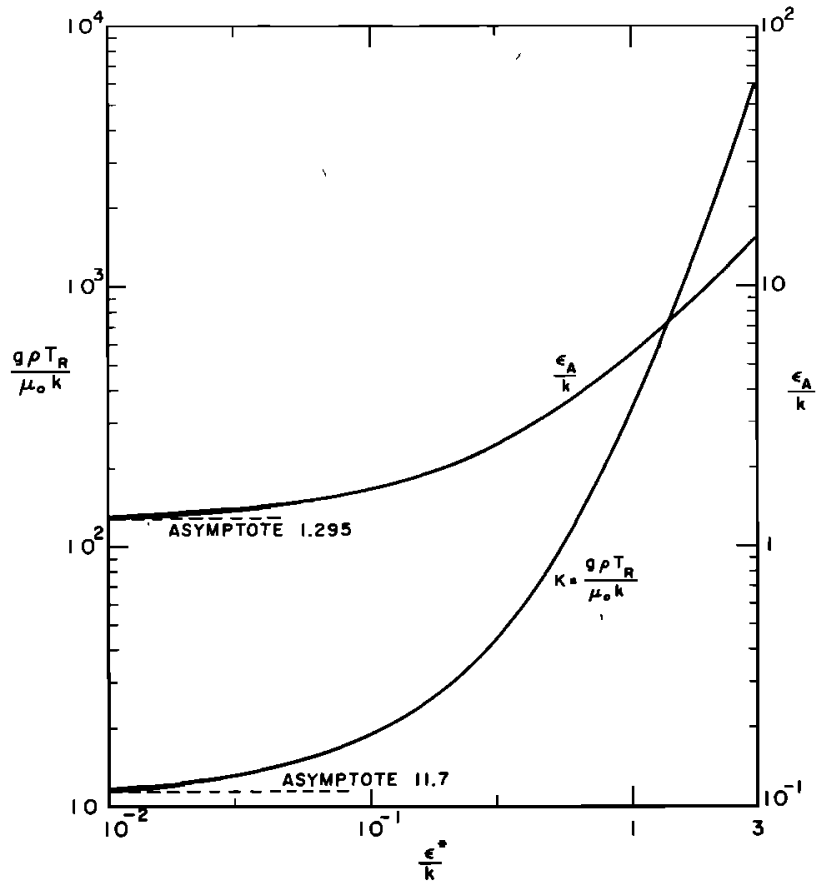

Fig. 5. Theoretical dependence of the parameter $K$ and apparant increase of viscosity with depth (given by $\epsilon_{A}$ ) on the real increase of viscosity with depth (given by $\epsilon^{*}$ ).

variation will be termed the real viscosity variation in the mantle to distinguish it from the apparent variations in $\mu$ introduced through (32) because of the dependence of the strain rate $\dot{e}$ on position $z$. Substituting (33) and (31) into (32) yields

$$
\mu=\mu_{0}\left\{k \frac{\partial \eta}{\partial t}\right\}^{-2 / 3} \exp \left[-\epsilon^{*} z-\frac{2}{3}\left(\frac{\epsilon}{2}+\beta\right) z\right]
$$

But the original premise of the solution was that

$$
\mu=\mu_{0} e^{-\epsilon z}
$$

and hence comparison of the exponents requires that

$$
\epsilon=3 \epsilon^{*} / 2+\beta
$$

It follows from writing (35) as

$$
\mu=\mu_{0} \exp \left(-\epsilon^{*} z-\epsilon_{A} z\right)
$$

that $\epsilon_{A}=\left(\epsilon-\epsilon^{*}\right)$ is the apparent increase in viscosity with depth due to strain rate decrease.

Examine first the simpler case of no real viscosity increase or $\epsilon^{*}=0$. It follows that $\beta / \epsilon=1$ and thence from the definition of $\beta$ (equation (16)) that $\epsilon=\epsilon_{A}=1.295 k$. Thus the apparent viscosity variation is a simple function of wave number. Further, in this simple case, from the definition $(27 b), K$ becomes 11.7 , and hence the relaxation time $T_{R}$ and surface viscosity $\mu_{0}$, which are now functions of time, are related at any instant by $T_{R}=11.7 \mu_{0} \mathrm{k} / \mathrm{g} \rho$ or $\mu_{0}=0.085 \mathrm{g \rho} T_{R} / \mathrm{k}$.

When a nonzero value of the real viscosity variation $\epsilon^{*}$ is included in the picture, it is necessary to solve the simultaneous equations (16) and (36) for $\epsilon$ and $\beta$ for given values of $\epsilon^{*}$ and $k$. Thus the values of both $\epsilon_{A} / k$ and $K$ are functions of $\epsilon^{*} / k$; these relationships are presented graphically in Figure 5 . It is particularly important to note that the apparent viscosity increase $\epsilon_{A}$ is always greater than the real variation $\epsilon^{*}$. Hence isostatic recovery flows with strain rates sufficiently great for disloca- tion glide to be the flow process will always be dominated by the apparent viscosity increase with depth $\epsilon_{A}$ rather than any real variation $\epsilon^{*}$.

It follows that having obtained $K$ for a particular isostatic recovery flow we may write

$$
\mu_{0}=g \rho T_{R} / k K
$$

and calculate values of $\mu_{0}$ at various times during the motion, knowing the corresponding value of $T_{R}$ at that instant. Further, we shall rather arbitrarily take a value of $-(k / 4)(\partial \eta / \partial t)_{z=0}$ as representing the mean surface strain rate $\dot{e}_{0}$ over the uplifting area, that is to say, one quarter of the value at $z=0$. By definition of $T_{R}$ this can be written in the alternative form

$$
\dot{e}_{0}=-\frac{k}{4}\left(\frac{\partial \eta}{\partial t}\right)_{z=0}=\frac{k(\eta)_{z=0}}{4 T_{R}}
$$

where $(\eta)_{z=0}$ is the remaining uplift.

The philosophy of the next section is to evaluate corresponding viscosities and strain rates from (38) and (39) and in this way to compare the isostatic recovery data with Weertman's theory. This procedure is followed for a number of chosen values for the real viscosity variation $\epsilon^{*}$. The intention is to examine whether the isostatic recovery data are compatible with Weertman's theory and, if they are, to determine which value of the real viscosity increase $\epsilon^{*}$ yields the greatest degree of consistency and agreement.

But before proceeding with this it is worth noting that since $\mu_{0}$ is now a function of time, the integration of (27a) in order to determine the deformation $\eta(t)$ no longer leads to exponential decay of the remaining uplift. In fact, from (34) the following is obtained:

$$
\mu_{0}=\mu_{0} *\left(k \frac{\partial \eta}{\partial t}\right)^{-2 / 3}
$$

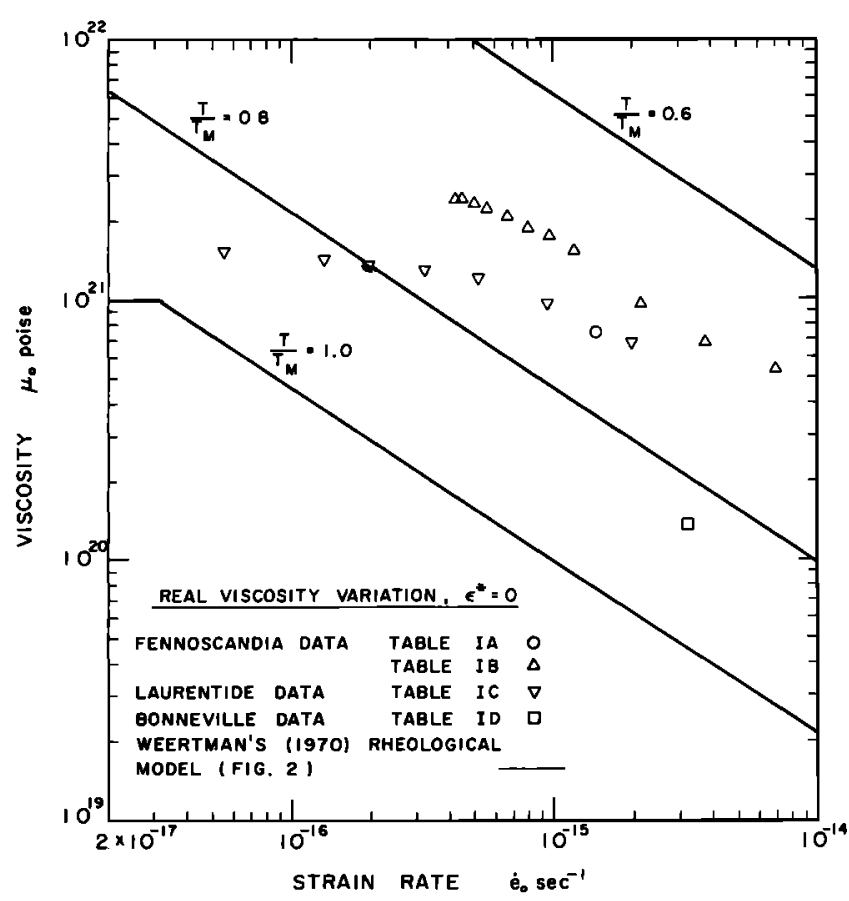

Fig. 6. Comparison between Weertman's rheological model for the mantle and data derived from the isostatic recovery information with the assumption that the real increase of viscosity with depth is zero $\left(\epsilon^{*}=0\right)$. Compare Figures 6,7 , and 8 . 


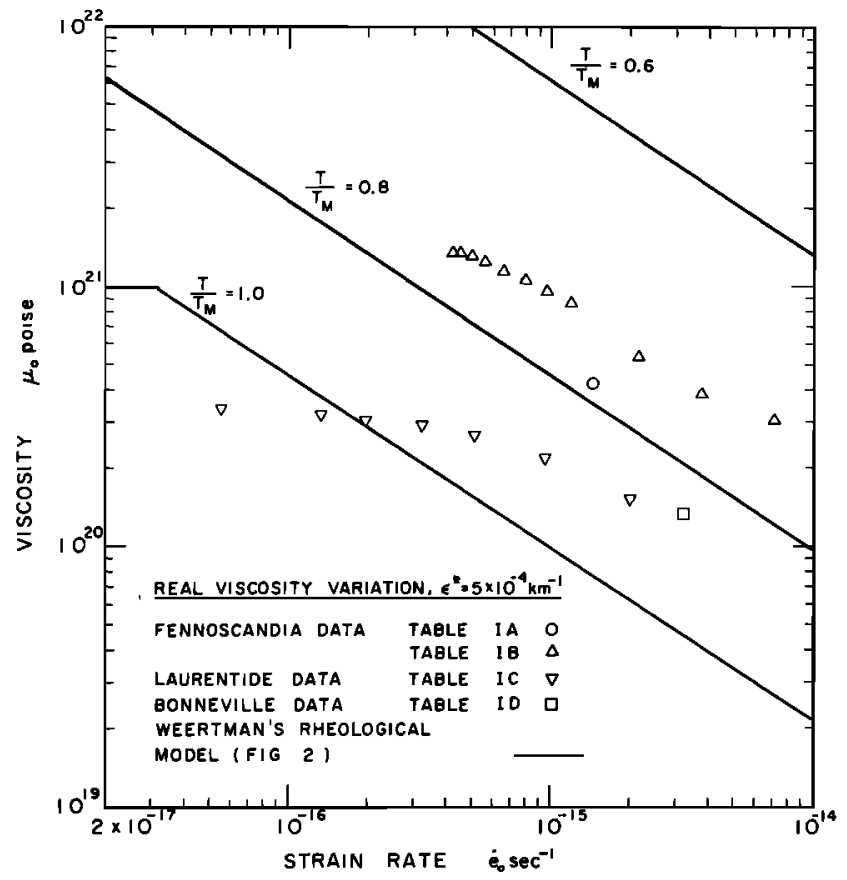

Fig. 7. A comparison between Weertman's rheological model for the mantle and data derived from the isostatic recovery information, with the assumption that the real increase of viscosity with depth is given by $\epsilon^{*}=5 \times 10^{-4} \mathrm{~km}^{-1}$.

If one substitutes in (27a) and integrates, the recovery flow is such that the remaining uplift varies with time as

$$
\eta=\frac{\eta_{0}}{[1+(t / T)]^{1 / 2}}
$$

where $\eta_{0}$ is the intitıal displacement at $t=0$, and

$$
T=\frac{1}{2\left(k \eta_{0}\right)^{2}}\left(\frac{\mu_{0}^{*} K}{g \rho}\right)^{3}
$$

It also follows that the relaxation time $T_{R}$ should vary with time as $T_{R}=2 T+t$.

\section{Comparison of Isostatic Recovery Data With WEERTMAN'S THEORY}

Isostatic recovery data from McConnell [1968] and Heiskanen and Vening Meinesz [1958] for the Fennoscandian ice sheet, from Andrews [1970] for the Laurentide ice sheet, and from Crittenden [1963] for pluvial Lake Bonneville are presented in Table 1. Each of these sets requires some additional comment. First, it must be noted that since the fluid is now taken to be nonlinear with a non-Newtonian strain rate dependent viscosity, the law of superposition no longer holds. Hence it is not valid to synthesize a particular motion from a series of Fourier components of different wave numbers $k$ in the manner of McConnell [1968]. However, since there is clearly a dominant wave number of $k=4 \times 10^{-3}$ in McConnell's data, it does seem justifiable to select this point for analysis, as representing the entire motion quite closely. The same dominant wave number is assumed to be relevant to the Fennoscandian data of Heiskanen and Vening Meinesz [1958] (Table 1). In Table 1 the remaining uplift is extracted from McConnell's data, and the remaining uplift is calculated from Andrews's [1970] percentage figures and his total maximum uplift of $450 \mathrm{~m}$; the wave number $k$ is based on the mean dimension, $3400 \mathrm{~km}$, of the ice sheet (see the section on isostatic recovery data). In the Bonneville data (Table 1 ), $k$ is again based on the dimension $L=200 \mathrm{~km}$. Then the strain rates $\dot{e}_{0}$ are computed from (39) and presented in column 4, and viscosities $\mu_{0}$ are computed from (38) by using Figure 5 and three different values for the real viscosity increase $\epsilon^{*}$, namely, $0.5 \times 10^{-4}$ and $24 \times 10^{-4} \mathrm{~km}^{-1}$. These data are presented graphically in Figures 6, 7, and 8. Comparison is made with the theoretical model of Weertman (Figure 2), which is also depicted in these figures. Now, it is fairly well accepted [Jacobs, 1956] that the temperature $T$ in the upper mantle is close to the melting temperature $T_{M}$, so that in the region where most of the flow occurs, $T / T_{M} \approx 1$. Hence we anticipate that the isostatic recovery data ought to correspond most closely with Weertman's line for $T / T_{M}=1$. But it should be borne in mind in making such a comparison that Weertman's rheological model can only be regarded as predicting actual values to within perhaps an order of magnitude.

Consider first the data for $\epsilon^{*}=0$ shown in Figure 6 . The two major data sets have a characteristic shape that is quite consistent with the shape of the theoretical lines. They do, however, appear consistent with a rather low value of $T / T_{M}$. Now when a small value of $\epsilon^{*}=5 \times 10^{-4} \mathrm{~km}^{-1}$ is introduced in Figure 7 , the Bonneville data become more consistent with the rest, and the data correspond with a more acceptable and higher value of $T / T_{M}$. This value is indicated as about 0.85 but in the light of the above comment on the accuracy of the theory may be regarded as being of the order of unity. However, further increase of $\epsilon^{*}$ to $24 \times 10^{-4} \mathrm{~km}^{-1}$ leads to more widely scattered data and lack of compatibility with the theory.

Thus the analysis of the isostatic recovery data presented here appears to yield the most consistent results and to be in closest agreement with the Weertman theory when the real increase of viscosity with depth is rather small and of a magnitude represented by an exponential function of $5 \times 10^{-4} z$,

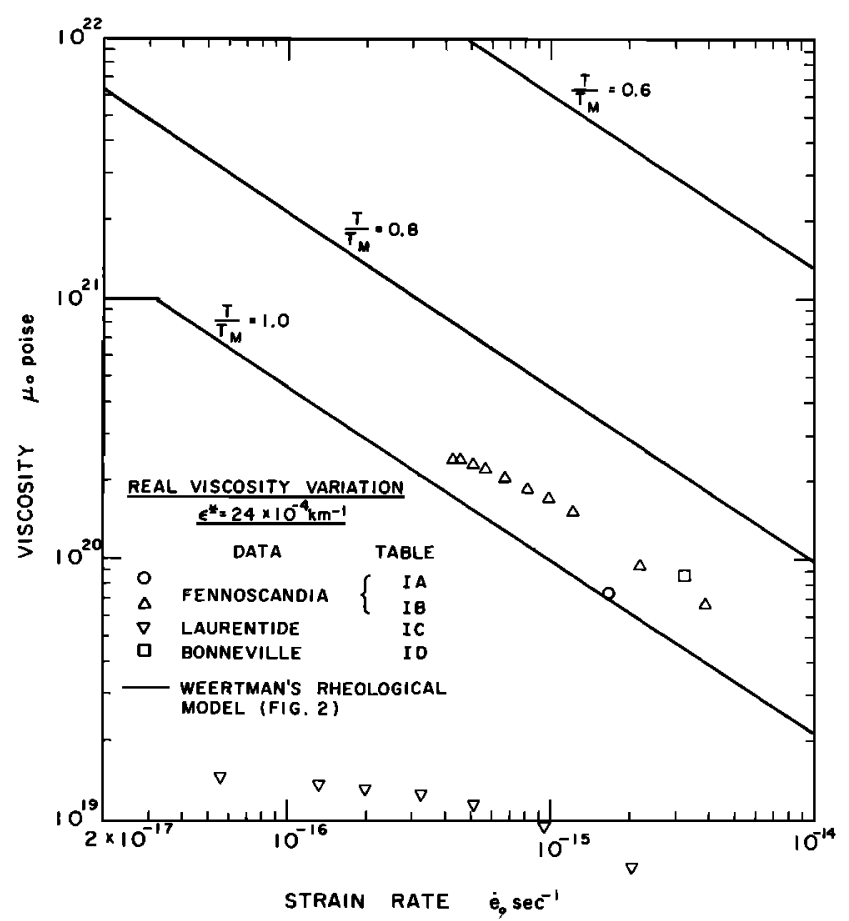

Fig. 8. A comparison between Weertman's rheological model for the mantle and data derived from the isostatic recovery information, with the assumption that the real increase of viscosity with depth is given by $\epsilon^{*}=24 \times 10^{-4} \mathrm{~km}^{-1}$. 


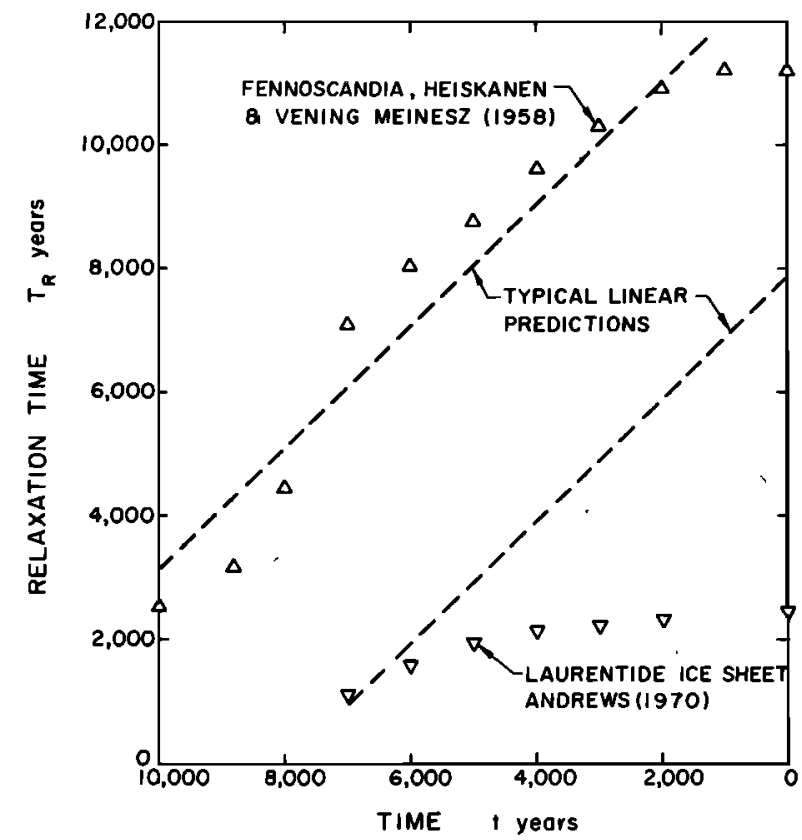

Fig. 9. Variation of the relaxation time $T_{R}$ with time $t$ and comparison with typical curves of the predicted linear dependence. For the Fennoscandia data, $t$ is in years B.P.; for the Laurentide data, $t$ is evaluated from the time since deglaciation.

wheré $z$ is the depth in kilometers. This variation is shown in Figure 1 and is now consistent with the more recent analyses of viscosity variation in the mantle discussed in the introduction.

Finally, by way of a further check on the validity of the present analysis we should also compare the data of Table 1 with the predicted linear (or near-linear) variation of the relaxation time $T_{R}$ with time $t$ suggested at the end of the previous section. This comparison is effected in Figure 9, where the Fennoscandian data of Heiskanen and Vening Meinesz [1958] are in very fair agreement with the predicted linear variation. The Laurentide data are much poorer in this regard and indeed are more suggestive of a constant $T_{R}$. The comparison is, however, more difficult to evaluate precisely, since Andrews [1970] computes time from the moment of deglaciation, which can vary significantly from location to location. As far as Figures 6, 7, and 8 are concerned, it should, however, be noted that a hypothetical change in the observational data at the later times, which would increase the relaxation times to a value of about 8000 years in concert with that ex pected on the basis of the theory, would actually improve the agreement between the Laurentide and the other data by increasing the subsequent viscosities and decreasing the strain rates.

\section{Conclusions}

This paper has demoristrated that the increase of viscosity with depth within the earth's mantle predicted by previous analyses of isostatic recovery, such as the analysis of McConnell [1968], is most probably an artifice caused by the decrease of strain rate with depth inherent in such flows. It is shown that the strain rates are sufficiently large for dislocation glide to be the flow process rather than diffusion creep. Then accepting Weertman's [1970] theory that the viscosity under these circumstances decreases with increasing strain rate, one must distinguish between the real variation of viscosity with depth and an apparent increase due to decreasing strain rate in isostatic recovery flows. A hydrodynamic analysis of such a flow is present in the section on isostatic recovery flow in mantle of nonuniform viscosity and in the section on strain rates and isostatic recovery flows and is used to reexamine some isostatic recovery data in this new light. Observations on the Fennoscandian and Laurentide ice sheets and pluvial Lake Bonneville are converted to strain rates and viscosities at a reference point and compared with Weertman's [1970] rheological model for the mantle. Though the numerical accuracy of Weertman's rheological model is somewhat limited, the observational data-appear to be most self-consistent and to agree most closely with the theory when the real increase of viscosity is an exponential function with an argument of the order of $5 \times 10^{-4} z$, where $z$ is the depth in kilometers. Such an increase of viscosity with depth is much smaller than previous suggestions based on what is now claimed to be an erroneous interpretation of the isostatic recovery data by McConnell [1968] and others (order of $6 \times$ $10^{-3} z$ ). In general, the analysis and this real variation of viscosity with depth appear to be consistent with Weertman's [1970] model of the mantle and to provide some verification of such a model.

Addendum. Following completion of this manuscript Post and Griggs [1973] published a paper in which isostatic recovery in a non-Newtonian mantle was discussed in terms of gross strain rates and stresses characterizing the flow. Their conclusion based on evaluation of Fennoscandian data suggests a value of the non-Newtonian index $n$ close to 3 , which is in agreement with character of Weertman's dislocation glide and therefore with one of the general conclusions of the present paper.

\section{REFERENCES}

Andrews, J. T., A Geomorphological Study of Post-Glacial Uplift With Particular Reference to Arctic Canada, Institute of British Geographers, London, 1970.

Chandrasekhar, S., Hydrodynamic and Hydromagnetic Stability, Clarendon, Oxford, 1961.

Crittenden, M. D., Effective viscosity of the earth derived from isostatic loading of Pleistocene Lake Bonneville, J. Geophys. Res., 68(19), 5517-5530, 1963.

Dicke, R. H., Average acceleration of the earth's rotation and the viscosity of the deep mantle, J. Geophys. Res.. 74(25), 5895-5902, 1969.

Goldreich, P., and A. Toomre, Some remarks on polar wandering, $J$. Geophys. Res., 74(10), 2555, 1969.

Gordon, R. B., Diffusion creep in the earth's mantle, J. Geophys. Res., 70(10), 2413-2418, 1965.

Gordon, R. B., Thermally activated processes in the earth: Creep and seismic attenuation, Geophys. J., 13, 33, 1967.

Haskell, N. A., The motion of a viscous fluid under a surface load, 1, Physics, 6(8), 265-269, 1935.

Haskell, N. A., The motion of a viscous fluid under a surface load, 2, Physics, 7(2), 56-61, 1936.

Haskell, N. A., The viscosity of the asthenosphere, Amer. J. Sci., 33(193), 22-28, 1937.

Heiskanen, W. A., and F. A. Vening Meinesz, The Earth and Its Gravity Field, McGraw-Hill, New York, 1958.

Jacobs, J. A., The earth's interior, in Handbuch der Physik, Geophysik l, edited by J. Bartets, p. 364, Springer, New York, 1956.

Jeffreys, H., On the hydrostatic theory of the figure of the earth, Geophys. J., 8, 196-202, 1952.

Macdonald, G. J. F., The internal constitutions of the inner planets and the moon, Space Sci. Rev., 2, 273, 1963.

McConnell, R. K., Viscosity of the mantle from relaxation time spectra of isostatic adjustment, $J$. Geophys. Res., 73(22), 7089-7105, 1968.

Milne Thomson, L. M., Theoretical Hydrodynamics, Macmillan, New York, 1968. 
Niskanen, E., On the viscosity of the earth's interior and crust, $A n n$. Acad. Sci. Fenn., Ser. A, 3(15), 22, 1948.

Post, R. L., and D. T. Griggs, The earth's mantle: Evidence of nonNewtonian flow, Science, 181(4106), 1242, 1973.

Takeuchi, M., Time scales of isostatic compensations, J. Geophys. Res., 68(8), 2357, 1963.

Takeuchi, M., and S. Sakata, Convection in a mantle with variable viscosity, J. Geophys. Res., 75(5), 921-927, 1970.
Vening Meinesz, F. A., The determination of the earth's plasticity from postglacial uplift of Scandinavia: Isostatic adjustment, Proc. Kon. Ned. Akad. Wetensch., 40, 654-662, 1937.

Weertman, J., The creep strength of the earth's mantle, Rev. Geophys. Space Phys., 8, 145-168, 1970.

(Received December 6, 1973; accepted April 22, 1974.) 